\title{
Spiral Gall
}

\author{
Murray McKeich \\ RMIT University \\ Melbourne, Australia \\ Murray.mckeich@rmit.edu.au
}

\section{INTRODUCTION}

Spiral Gall is a single channel HD (1080p) video work that uses video footage of wind blown tree foliage to animate the speaking heads of surreal beings (see Figure 5). The subtle complexities of natural movement in the video footage are transferred to the constructed (digital photo montage) faces via a process that uses time-based shifts in the videos tonal density to animate facial movement. The dislocation of movement from video to animation imbues the animated beings with a complex and surreal simulation of life that is natural but inhuman.

\section{PROCESS}

The Spiral Gall video work is produced using a range of generative systems and strategies that began with videoing windblown tree foliage (Figure 1).

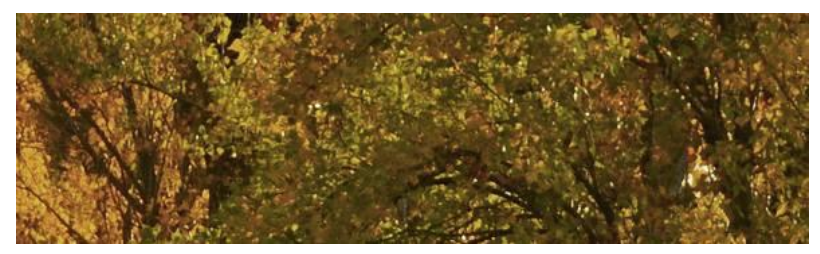

Figure 1: Wind blown tree foliage video (still, detail)

I then collected windfall stems infested with Black Poplar Spiral Galls from the same location (Figure 2).

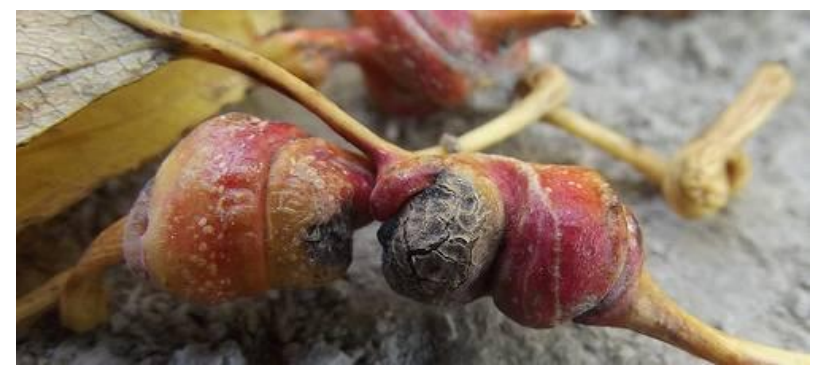

Figure 2: Black Poplar Spiral Galls
Individual galls are placed on a flatbed scanner and scanned to form an image library (Figure 3). Via generative collage processes, these image elements are combined to form a diverse population of surreal 'beings' (see Figure 5).

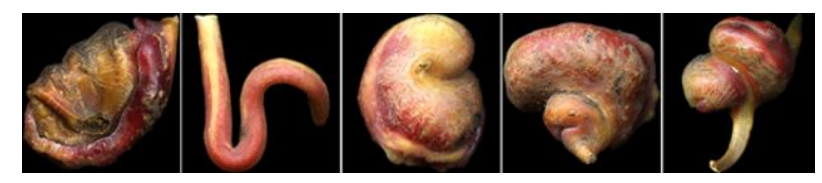

Figure 3: image library (sample)

The video footage of windblown tree foliage (Figure 1) is sampled down to form a pixilated grid (Figure 4).

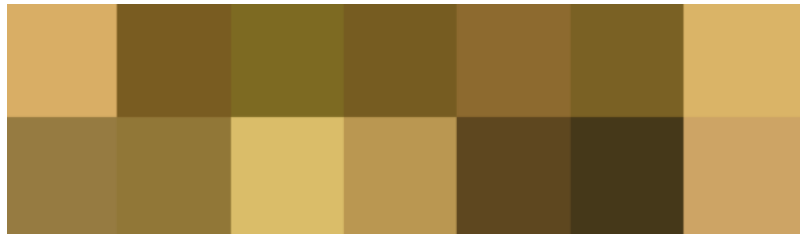

Figure 4: Pixelated grid video footage (still, detail)

Tonal shifts in each grid square of the footage are selectively used to animate various facial elements of the animated heads.

\section{DISCUSSION}

The representation of natural phenomena in generative computer art is dominated by the use of code-based simulations (e.g., Evolutionary Art). However, this often requires a challenging degree of coding skill. By contrast, the processes I have developed can simulate complex natural phenomena in an artwork by using relatively simple filter tools and processes found in the off-the-shelf software package Adobe After Effects. This methodology allows creative problems to be solved via visual means as opposed to the text-based semantics of computer code. The relative simplicity of this approach offers the potential for a visual artist to explore creative ideas with a high degree of efficiency and reflexive intuition. 


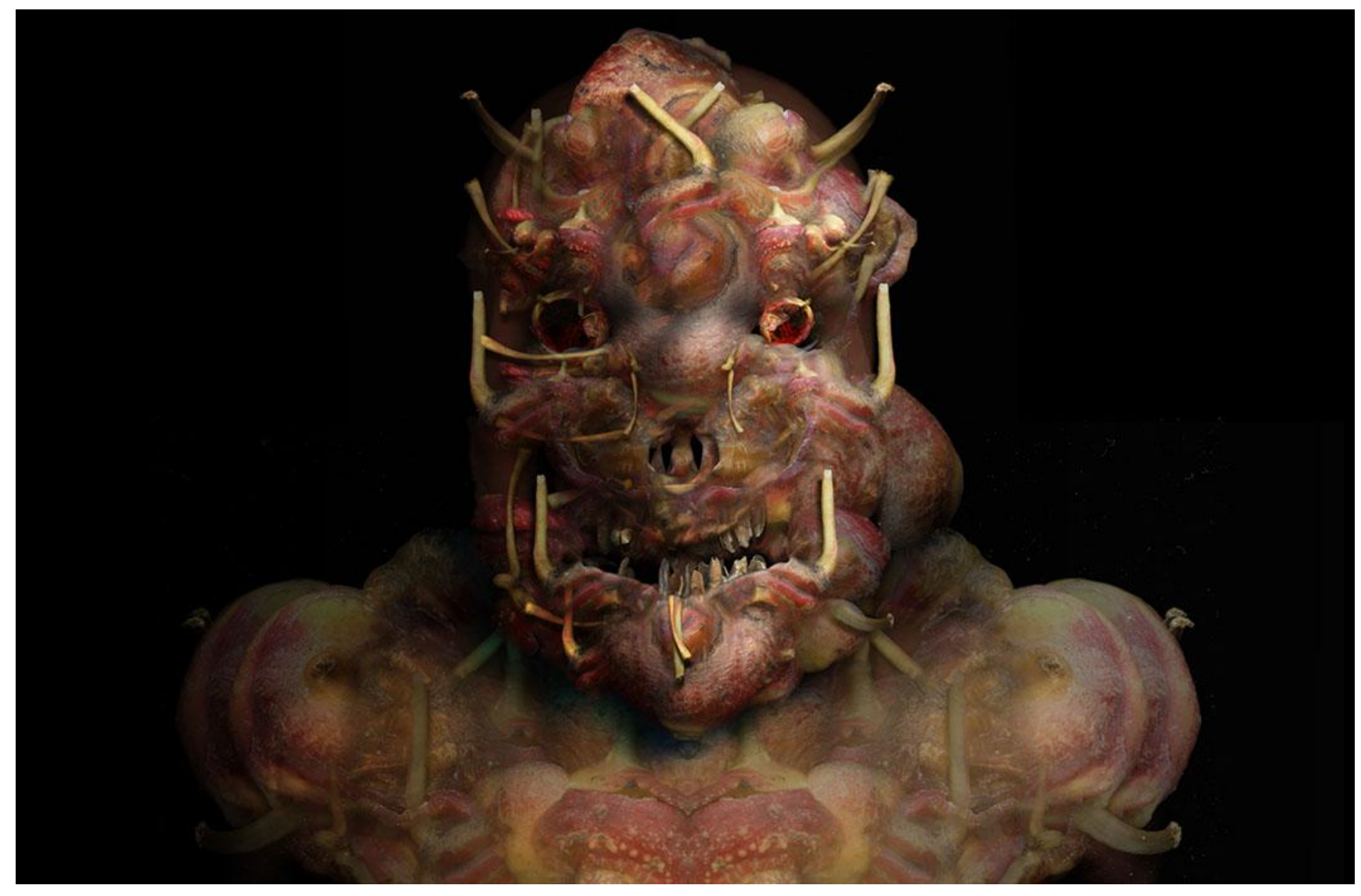

Figure 5: Video still from 'Spiral Gall' video (1080p) 